\title{
A FISIOTERAPIA EM GRUPO NO FORMATO DE CIRCUITO PODE MELHORAR A VELOCIDADE DA MARCHA DE PACIENTES COM DOENÇA DE PARKINSON?
}

Fabiana Araújo Silva ${ }^{1}$, Larissa Borba André ${ }^{2}$, Carla de Oliveira Carletti ${ }^{2}$, Andressa Sampaio Pereira ${ }^{2}$, Katiane Mayara Guerrero ${ }^{2}$, Thayná Araújo Maiolini Costa $^{3}$, Valesca Chioca Caiares ${ }^{3}$, Ana Flávia Balotari Botta Heloísa Balotari Valente ${ }^{4}$, Helder dos Santos Lopes ${ }^{4}$, Ygor de Matos Luciano ${ }^{4}$, Lúcia Martins Barbatto ${ }^{4}$, Augusto Cesinando de Carvalho ${ }^{4}$.

Universidade Estadual Paulista - UNESP; ${ }^{1}$ Programa de Pós-Graduação em Ciências da Motricidade Interunidades, Bauru, SP; ${ }^{2}$ Programa de Residência em Saúde - Área Reabilitação Física; ${ }^{3}$ Pós-Graduação Lato Sensu em Fisioterapia; ${ }^{4}$ Curso de Fisioterapia da FCT, Presidente Prudente, SP. E- mail: lari borba@hotmail.com

\section{RESUMO}

A Doença de Parkinson (DP) é caracterizada pela degeneração progressiva de neurônios dopaminérgicos da substância negra, resultando em desordens não motoras e motoras que estão relacionadas com o aparecimento de tremor de repouso, bradicinesia, rigidez articular, instabilidade postural, alterações no padrão da marcha, declínio do equilíbrio. A Fisioterapia em Grupo no formato de Circuit Training (FGCT) é um modelo terapêutico baseado em estações de trabalho dispostos num formato de circuito dirigido que reproduzem atividades físicas. O objetivo foi avaliar a velocidade de marcha rápida, de indivíduos com DP submetidos a sessões de FGTC. Participaram deste estudo 13 voluntários com DP. A avaliação inicial (AV1) foi realizada utilizando o Time up and go (TUG) e o Teste de velocidade de marcha de 10 metros (TV10M). No TUG, a velocidade de execução média foi de 0,68 $\pm 0,19 \mathrm{~m} / \mathrm{seg}$ na AV1 e 0,66 $\pm 0,14 \mathrm{~m} / \mathrm{seg}$ na AV2 e no TV10M de 1,36 $\pm 0,26 \mathrm{~m} / \mathrm{seg}$ na AV1 enquanto na AV2 foi $1,45 \pm 0,32 \mathrm{~m} / \mathrm{seg}$, sem diferença significante entre os dois momentos de medida. Os parkinsonianos não apresentaram melhoras funcionais talvez porque a terapia não teve exercícios com enfoque na velocidade da marcha e o tempo de terapêutica pode não ter sido suficiente para ocorrer mudanças nesse aspecto. Porém, sabe-se que a reabilitação em grupo contribui na melhora do estado de saúde global do paciente, permitindo maior socialização entre os indivíduos. Pode-se concluir que o protocolo terapêutico utilizado não foi suficiente para alterar a velocidade da marcha dos pacientes.

Palavras-chave: Doença de Parkinson, marcha, exercícios em circuito, Fisioterapia, reabilitação neurológica.

\section{CAN GROUP PHYSIOTHERAPY IN CIRCUIT FORMAT IMPROVE THE SPEED OF THE GAIT OF PATIENTS WITH PARKINSON DISEASE?}

\begin{abstract}
Parkinson's disease (PD) is characterized by the progressive degeneration of dopaminergic neurons of the substantia nigra, resulting in non-motor and motor disorders that are related to the appearance of resting tremor, bradykinesia, joint stiffness, postural instability, changes in gait patterna and decline in equilibrium. Group Physiotherapy in Circuit Training (FGCT) format is a therapeutic model based on workstations arranged in a directed circuit format that reproduce physical activities. The objective was to evaluate the fast walking speed of individuals with PD submitted to FGTC sessions. Thirteen volunteers with PD participated in this study. The initial assessment (AV1) was performed using the Time Up and Go (TUG) and the 10-Meter Speed Test (TV10M). In the TUG, the average execution speed was $0.68 \pm 0.19 \mathrm{~m} / \mathrm{seg}$ in AV1 and $0.66 \pm 0.14 \mathrm{~m} / \mathrm{seg}$ AV2 and in TV10M of $1.36 \pm 0.26 \mathrm{~m} / \mathrm{seg}$ in AV1 while in AV2 it was $1.45 \pm 0.32$ $\mathrm{m} / \mathrm{seg}$, with no significant difference between the two measurement moments. Parkinsonians did not show functional improvements, perhaps because the therapy did not exercise with focus on gait speed, and the time of therapy may not have been sufficient for changes in this aspect. However, it is known that group rehabilitation contributes to the improvement of the patient's overall health, allowing greater socialization among individuals. It can be concluded that the therapeutic protocol used was not enough to alter the gait speed of the patients.
\end{abstract}


Keywords: Parkinson Disease, gait, circuit-based exercise, physical therapy specialty, neurological rehabilitation.

\section{INTRODUÇÃO}

A Doença de Parkinson (DP) é caracterizada pela degeneração progressiva de neurônios dopaminérgicos da substância negra, resultando em desordens não motoras e motoras $^{1}$ que estão relacionados com 0 aparecimento de tremor de repouso, bradicinesia, rigidez articular, instabilidade postural $^{2}$, alterações no padrão da marcha, declínio do equilíbrio, dificuldades em realizar transferências e aumento do risco de quedas, comprometendo a mobilidade e participação social desses indivíduos ${ }^{3}$. A DP acomete cerca de 1 a $3 \%$ da população acima de 65 anos e no Brasil a prevalência é de $3,3 \%{ }^{4}$ e, portanto, é um grave problema de saúde pública.

As desordens observadas na marcha e no equilíbrio tem se apresentado como os principais objetivos terapêuticos, sendo que nas fases iniciais da doença são pouco incapacitantes, agravando-se com a progressão da mesma ${ }^{5}$ e por isso terapêuticas que visam amenizar estes sinais e sintomas são importantes em todas as fases da doença.

A marcha do parkinsoniano apresenta o padrão de rigidez, hipocinesia e diminuição na velocidade, com redução da amplitude do passo, porém sem alteração ou discreto aumento da cadência. Os distúrbios posturais caracterizados por flexão de tronco, hipertonia e movimento reduzido do quadril, também contribuem para a alteração na marcha, assim como a festinação, descrita como pequenos passos rápidos feitos na tentativa de manter o centro de gravidade entre os pés ${ }^{5}$.

Outra característica da DP é o freezing, caracterizado por uma interrupção transitória episódica, que dura alguns segundos, fazendo com o que indivíduo tenha dificuldade de avançar $^{6}$. Esse evento ocorre por estímulos visuais ou auditivos, assim como situações de estresse, ansiedade e depressão, podendo provocar a queda ${ }^{5}$.

O tratamento farmacológico utilizado na clínica médica especializada e os exercícios motores funcionais não são capazes de impedir a progressão da doença, porém contribuem para a redução na taxa de mortalidade e morbidade além da prevenção de complicações secundárias ${ }^{5,7}$.

O treinamento fisioterapêutico de parkinsonianos deve ser focado na prática de tarefas funcionais e em atividades que contribuam com a coordenação, equilíbrio, destreza, treino de marcha com variação da velocidade, melhora das transferências, da mobilidade articular, da extensibilidade e força muscular ${ }^{7}$. É necessário também que os programas terapêuticos associem agilidade sensório-motora, tarefas cognitivas e utilização de sequências mais desafiadoras ${ }^{8-10}$.

A reabilitação em grupo auxilia na melhora do estado de saúde global do paciente e aumenta a socialização entre os indivíduos ${ }^{5,7,8}$ e a Fisioterapia em Grupo no formato de Circuit Training (FGCT) é um modelo terapêutico baseado em estações de trabalho dispostos num formato de circuito dirigido que reproduzem atividades físicas como, caminhar, subir escadas, manter equilíbrio, com o objetivo de aprendizagem motora e retenção da tarefa ${ }^{11}$ sendo considerada uma boa opção de tratamento, pois além de proporcionar a prática intensiva das necessidades individuais; pode englobar dinâmicas de grupo que auxiliam na interação social que promove benefícios psicossociais, melhora do humor e da autoconfiança.

Os efeitos da FGCT foram descritos para pacientes com acidente vascular cerebral (AVC) e com esclerose múltipla $(E M)^{11,12}$, porém não foi observada esta terapêutica para pacientes com DP. Esse tipo de abordagem possui características positivas e fundamentais em um programa de treinamento físico, pois as estações de trabalho permitem aos pacientes adequarem a intensidade da atividade segundo suas necessidades, permite utilizar melhor o tempo de terapia ativa contribuindo para a redução de custos para o sistema de saúde e a formação em circuito permite a dinâmica de grupo, que favorece a interação social e o apoio ${ }^{3,11,13}$.

O fisioterapeuta deve criar estratégias para melhorar a mobilidade de pacientes com DP 
utilizando atividades físicas funcionais específicas, independentemente da categoria terapêutica e desta forma estudos que avaliem o efeito da FGCT podem incrementar a prática fisioterapêutica.

O objetivo deste estudo foi avaliar a velocidade de marcha rápida, de indivíduos com DP submetidos a 30 sessões de FGTC.

\section{METODOLOGIA}

Participaram deste estudo pessoas com DP frequentadoras de um centro de atendimentos de Fisioterapia e Reabilitação da Universidade Estadual Paulista (UNESP), campus de Presidente Prudente. Os indivíduos foram informados sobre a pesquisa e assinaram o termo de consentimento livre e esclarecido aprovados pelo Comitê de Ética da FCT-UNESP (CAAE: 45076015.3.0000.5402).

Foram incluídos no estudo indivíduos com diagnóstico clínico de DP com Escala de Estágios de Incapacidade de Hoehn e Yahr $(H \& Y)^{12}$ entre 1 e 3 , ausência de déficits cognitivos avaliados pelo Mini-exame do Estado Mental ${ }^{14}$. Foram excluídas outras patologias neurológicas, reumáticas ou ortopédicas.

Uma entrevista individual foi realizada para coleta de dados pessoais e verificação dos critérios de elegibilidade e posteriormente foi realizada a avaliação inicial (AV1) utilizando os seguintes testes: 1) Time up and go (TUG) ${ }^{15}$ para avaliar a mobilidade funcional de idosos e a marcha, 2) Teste de velocidade de marcha de 10 metros (TV10M) para avaliar a velocidade da marcha ${ }^{16}$. Durante a realização destes testes foi solicitado que os pacientes os realizassem com o máximo de velocidade possível, sem correr e sem se colocar em risco de quedas. Todos os testes foram repetidos após 30 sessões (AV2).

O período de intervenção teve início após a AV1 e durou 30 sessões, sendo realizadas 2 sessões por semana, perfazendo um total de 15 semanas. As sessões tinham duração de 55 min e iniciava com a aferição da pressão arterial e entrega de um número correspondente a estação na qual o paciente iniciaria a FGCT. Em seguida, a sessão foi subdividida em três fases:

1) Fase Inicial - Aquecimento: realizado com alongamentos globais ativos de flexores, rotadores e flexores laterais de cervical e tronco, adutores, rotadores internos e externos de ombro, flexores e extensores de quadril e joelho, orientados por um fisioterapeuta, com duração de 20 minutos;

2) Fase intermediária - FGCT: diferentes tipos de exercícios funcionais específicos previamente montados em forma de circuito dirigido em 10 espaços diferentes e interligados, denominados estações, e realizados de forma ativa durante 2 min por estação. As estações estão descritas a seguir: Estação 1: movimentos de flexo-extensão do quadril e joelho alternadamente, posicionados os pés em dois steps empilhados; Estação 2: de frente para o painel de esferas e segurando um bastão, dar um passo à frente, alternadamente, tocando com o bastão a esfera central; Estação 3: movimentos cíclicos com as pernas, utilizando um circloergômetro; Estação 4: na posição de sedestação, frente a uma mesa, deslocar blocos os cilindros de madeira de uma superfície mais alta para uma mais baixa e assim sucessivamente; Estação 5: agachamento em cima de um step com apoio manual em uma barra; Estação 6: dar passos para frente e para trás. Passando para dentro e fora de um bambolê; Estação 7: sentado em uma cadeira, levantar, percorrer a distância de 3 metros em direção a um cone, dar a volta no cone e retornar em direção a cadeira e sentar; Estação 8: caminhar nas barras paralelas sobre uma reta com um pé a frente do outro e depois pular cones com marcha lateral; Estação 9: subir e descer uma escada e rampa com caneleiras de 0,5 Kg nos tornozelos; Estação 10: de frente para o painel de esferas e segurando um bastão, dar um passo para a lateral forma alternada. A cada movimento tocar com o bastão a esfera do lado oposto ao que deu o passo.

3) Fase Final - Foi apresentada a letra de uma música e também cinco passos de dança que representam uma tarefa cognitiva. Os passos de dança eram constituídos de movimentos amplos com rotações de tronco, movimentação ativa de membros superiores e inferiores enfatizando a coordenação e o equilíbrio, deslocamento de peso juntamente com a dança. Esta fase durou 15 $\min$.

$\mathrm{Na}$ análise estatística, para verificar a normalidade dos dados, foi utilizado o teste de Shapiro-Wilk e para comparar o momento pré e pós-intervenção foi utilizado o teste $t$ Student para amostras pareadas, considerando significante $o$ valor de $p<0,05$. O effect size foi calculado e classificado bem como a Mínima Mudança Detectável (MMD). Foi utilizado o 
software Statistical Software for Social Sciences (SPSS Inc. Chicago, IL) versão 18.0.

\section{RESULTADOS}

Foram avaliados 13 indivíduos parkinsonianos com idade média de 72,15 $\pm 8,46$ anos sendo seis indivíduos no estágio 1 ; um no estágio 1,5 ; dois no estágio 2 , três no estágio 2,5 e um no estágio 3 da Escala de Hoehn e Yahr ${ }^{12}$.

$\mathrm{Na}$ análise dos valores do TUG, os parkinsonianos demonstraram uma velocidade de execução média de 0,68 $\pm 0,19 \mathrm{~m} / \mathrm{seg}$ na AV1 e $0,66 \pm 0,14 \mathrm{~m} / \mathrm{seg}$ AV2 e no TV10M de 1,36 \pm $0,26 \mathrm{~m} / \mathrm{seg}$ na AV1 enquanto na AV2 foi $1,45 \pm$ $0,32 \mathrm{~m} / \mathrm{seg}$, sem diferença significante entre os dois momentos de medida.

Para as diferenças entre os grupos também foi calculado o effect size, pela fórmula de Cohen (d). As leituras das magnitudes foram realizadas como efeito insignificante $(>=0,00 \mathrm{a}$ $0,15)$; pequeno efeito $(>=0,15$ e $<0,40)$; médio efeito $(>=0,40$ e $<0,75)$; grande efeito $(>0,75)$. Esse conceito estatístico é traduzido normalmente por tamanho, dimensão ou magnitude do efeito e pode ser definido como o grau em que o fenômeno está presente na população, isto é, a diferença efetiva na população. Assim, quanto maior for o ES, maior será a manifestação do fenômeno na população. No TUG, o efeito foi classificado como insignificante e no TV10m, pequeno efeito ${ }^{17}$.

A MMD no TV10m para parkinsonianos com idade média de 71 anos, fase média de $\mathrm{H} \& \mathrm{Y}$ 2 (podendo variar de 1 a 4$)^{18}$ é de $0,25 \mathrm{~m} / \mathrm{seg}$ sendo que a do presente estudo foi $0,09 \mathrm{~m} / \mathrm{seg}$. Não há valores definidos de MMD para TUG nesta população.

\section{DISCUSSÃO}

As fases iniciais da doença de Parkinson (estágios 1 e 2 de Hoehn e Yahr) são caracterizadas por alterações motoras e posturais predominantemente unilaterais, sendo assim pouco incapacitantes. Contudo, à medida que o estágio da doença progride, os sintomas físicos se agravam, tornando-se bilaterais, levando a uma diminuição da capacidade funcional ${ }^{19}$. A progressão da doença ocorre independentemente de qualquer fator, porém o tratamento medicamentoso associado com a prática de exercício físico e fisioterapia tem sido benéfico para a manutenção de algumas habilidades desses pacientes $5^{5,7,20}$.
Os participantes deste estudo, segundo a Escala de Hoehn e Yahr, estão classificados entre os estágios 1 e 3 estando portanto a maioria no estágio mais brando da doença. As avaliações utilizando o TUG e TV10M ${ }^{15,16}$ classificaram suas marchas como comunitária e independente, podendo inferir que a FGCT pode influenciar na manutenção desta condição funcional neste período avaliado.

O treinamento de parkinsonianos deve ser focado em prática de tarefas funcionais repetitivas, dessa forma, a FGCT tem se demonstrado uma abordagem terapêutica relevante, pois propicia um grande número de repetições de tarefa ou movimentos necessários que pode resultar em alterações neurais duradouras e melhorar a vida funcional desses indivíduos, e assim, influenciar a qualidade de vida $^{21,22}$.

A FGCT promove agilidade sensóriomotora, envolvendo coordenação, equilíbrio, destreza e treino de marcha, associação de tarefa dupla e utilizando sequências mais desafiadoras $^{7,8}$, tornando-a capaz de promover maior grau de independência em diversas atividades ${ }^{11}$.

A DP ocasiona uma alteração no padrão da marcha levando a diminuição do comprimento do passo. Dessa forma, o treinamento físico realizado de forma progressiva e orientada, pode ser eficaz no aumento da velocidade da marcha e distância percorrida ${ }^{5}$.

Embora os resultados deste trabalho não tenham demonstrado alterações motoras funcionais significantes e baixo effect size não se pode desconsiderar que a reabilitação em grupo contribui na melhora do estado de saúde global do paciente, permitindo maior socialização entre os indivíduos. O número de participantes, o tempo de duração e o pouco enfoque na velocidade da marcha pode ter influenciado os resultados deste trabalho. Apesar de não ser objeto deste trabalho, pode-se observar que parkinsonianos atendidos durante a execução deste trabalho mostraram-se bastante motivados e satisfeitos com a terapia, participando efetivamente durante as tarefas propostas e havendo maior interação entre os participantes $^{7,9,20}$.

\section{CONCLUSÃO}

O protocolo terapêutico utilizado não alterou a velocidade da marcha dos pacientes. 


\section{DECLARAÇÃO DE CONFLITO DE INTERESSE}

Os autores declaram não haver qualquer conflito de interesse que possa interferir na imparcialidade deste trabalho científico.

\section{REFERÊNCIAS}

1. Hallett M. Parkinson revisited: pathophysiology of motor signs. Adv Neurol. 2003;91:19-28.

2. Pereira JAC, Gaiad TP, Santos AP. Efeito do treinamento em circuito orientado para tarefa sobre o desempenho motor e a qualidade de vida de pacientes com doença de Parkinson: um estudo de casos. Rev Ter Ocup Univ São Paulo. 2016;27(3):329-35. DOI: http://dx.doi.org/10.11606/issn.22386149.v27i3p329-335

3. van den Heuvel MR, Kwakkel G, Beek PJ, Berendse HW, Daffertshofer A, van Wegen EE. Effects of augmented visual feedback during balance training in Parkinson's disease: a pilot randomized clinical trial. Parkinsonism Relat Disord. 2014;20(12):1352-8. DOI: https://doi.org/10.1016/j.parkreldis.2014.09.022

4. Barbosa MT, Caramelli P, Maia DP, Cunningham MC, Guerra HL, Lima-Costa MF, et al. Parkinsonism and Parkinson's disease in the elderly: a community-based survey in Brazil (the Bambui study). Movem Disord. 2006;21(6):800-8. DOI:

http://doi.org/10.1002/mds.20806

5. Grabli D, Karachi C, Welter ML, Lau B, Hirsch EC, Vidailhet $M$, et al. Normal and pathological gait: what we learn from Parkinson's disease. J Neurol Neurosurg Psych. 2012;83(10):979-85. DOI: http://doi.org/10.1136/jnnp-2012-302263

6. Nutt JG, Bloem BR, Giladi N, Hallett M, Horak FB, Nieuwboer A. Freezing of gait: moving forward on a mysterious clinical phenomenon. Lancet Neurol 2011;10:734-44. DOI: http://doi.org/10.1016/S14744422(11)70143-0

7. Allen NE, Sherrington C, Suriyarachchi GD, Paul SS, Song J, Canning CG. Exercise and motor training in people with Parkinson's disease: a systematic review of participant characteristics, intervention delivery, retention rates, adherence, and adverse events in clinical trials. Parkinson's Dis. 2012;2012:854328. DOI: http://dx.doi.org/10.1155/2012/854328
8. de Dreu MJ, van der Wilk AS, Poppe E, Kwakkel G, van Wegen EE. Rehabilitation, exercise therapy and music in patients with Parkinson's disease: a metaanalysis of the effects of music-based movement therapy on walking ability, balance and quality of life. Parkinsonism Relat Disord. 2012;18(Suppl 1):S114-9. DOI: http://dx.doi.org/10.1016/S1353-8020(11)70036$\underline{0}$

9. Tomlinson CL, Patel S, Meek C, Herd CP, Clarke CE, Stowe $\mathrm{R}$, et al. Physiotherapy intervention in Parkinson's disease: systematic review and metaanalysis. $\mathrm{Br}$ Med J. 2012;345:e5004. DOI: https://doi.org/10.1136/bmj.e5004

10. Shu HF, Yang T, Yu SX, Huang HD, Jiang LL, Gu JW, et al. Aerobic exercise for Parkinson's disease: a systematic review and meta-analysis of randomized controlled trials. PloS one. 2014;9(7):e100503. DOI: http://dx.doi.org/10.1371/journal.pone.0100503

11. English CK, Hillier SL, Stiller KR, Warden-Flood A. Circuit class therapy versus individual physiotherapy sessions during inpatient stroke rehabilitation: a controlled trial. Arch Phys Med Rehab. 2007;88(8):955-63. DOI: http://dx.doi.org/10.1016/j.apmr.2007.04.010

12. Hoehn MM, Yahr MD. Parkinsonism: onset, progression, and mortality. 1967. Neurology. 2001; 57(10 Suppl 3):S11-26.

13. Dean CM, Richards CL, Malouin F. Task-related circuit training improves performance of locomotor tasks in chronic stroke: a randomized, controlled pilot trial. Arch Phys Med Rehab. 2000;81(4):409-17. DOI: http://dx.doi.org/10.1053/mr.2000.3839

14. Cockrell JR, Folstein MF. Mini-Mental State Examination (MMSE). Psychopharmacol bull. 1988;24(4):689-92.

15. Podsiadlo D, Richardson S. The timed "Up \& Go": a test of basic functional mobility for frail elderly persons. J Am Geriat Soc. 1991;39(2):142-8. DOI: http://dx.doi.org/10.1111/i.1532-

5415.1991.tb01616.x

16. Salbach NM, Mayo NF, Higgins J, Ahmed S, Finch LE, Richards CL. Responsiveness and predictability of gait speed and other disability measures in acute stroke. Arch Phys Med Rehabil. 2001;82(9):1204-12.

17. Loureiro LMJ, Gameiro MGH. Interpretação crítica dos resultados estatísticos: para lá da significância estatística. Rev Enferm Refer. 2011;3(3):151-62.

18. Steffen T, Seney M. Test-retest reliability and minimal detectable change on balance and ambulation tests, the 36-item short-form health survey, and the unified Parkinson disease rating scale in people with 
parkinsonism. Phys Ther. 2008;88(6):733-46. DOI: http://dx.doi.org/10.2522/ptj.20070214

19. Shanahan J, Morris ME, Bhriain ON, Saunders J, Clifford AM. Dance for People With Parkinson Disease: What Is the Evidence Telling Us? Arch Phys Med Rehabil. 2015;96(1):141-53.

DOI:

http://dx.doi.org/10.1016/i.apmr.2014.08.017

20. Lauzé M, Daneault JF, Duval C. The Effects of physical activity in Parkinson's Disease: a review. J Parkinsons Dis. 2016;6(4):685-98. DOI: http://dx.doi.org/10.3233/JPD-160790

21. Brogardh C, Lexell J. Effects of cardiorespiratory fitness and muscle-resistance training after stroke. Phys Med Rehab. 2012;4(11):901-7. DOI: http://dx.doi.org/10.1016/j.pmrj.2012.09.1157

22. Jeon BJ, Kim WH, Park EY. Effect of task-oriented training for people with stroke: a meta-analysis focused on repetitive or circuit training. Topics Stroke Rehab. 2015;22(1):34-43. DOI: http://dx.doi.org/10.1179/1074935714Z.0000000035

Recebido para publicação em 16/08/2017

Revisado em 09/09/2017

Aceito em 15/09/2017 